\title{
EVALUATION AND ANALYSIS OF MINIMUM PHONE ERROR TRAINING AND ITS MODIFIED VERSIONS FOR LARGE VOCABULARY MANDARIN SPEECH RECOGNITION
}

\author{
Yung-Jen Cheng, Che-Kuang Lin, and Lin-Shan Lee \\ Graduate Institute of Communication Engineering, National Taiwan University, Taipei \\ \{quezacot,kimchy\}@speech.ee.ntu.edu.tw,1slee@gate.sinica.edu.tw
}

\begin{abstract}
This paper reports a detailed study on Minimum Phone Error (MPE), Minimum Phone Frame Error (MPFE), and a physical-state level version of Minimum Bayes Risk (sMBR) training, as well as several modified versions of them, for transcription of large vocabulary Mandarin broadcast news. We found the results are quite different from these observed previously for English and Arabic broadcast news tasks[1], in particular the trends are different when different performance measures (word and character accuracies) are used. This makes the difference for Chinese language, for which character accuracy is usually more important, while word accuracy is commonly used for other languages. Modifications to these approaches tested here include considering the variable phone length and applying penalties to erroneous frames. They were shown to be able to significantly improve character accuracy in our experiments.
\end{abstract}

Index Terms - Discriminative training, Minimum Phone Error, Minimum Phone Frame Error, Minimum Bayes Risk

\section{INTRODUCTION}

There has been great interest in discriminative training techniques for speech recognition. Unlike conventional maximum likelihood (ML) training algorithms, discriminative training updates parameters considering the training based on not only the correct transcription but also the competing hypotheses obtained from either n-best lists or lattices, thus yielding more accurate models. Various criteria and objective functions have been proposed and shown effective for discriminative training, such as Maximum Mutual Information (MMI) [2] and Minimum Classification Error (MCE) [3]. Recently, Minimum Phone Error (MPE) training has been proposed and achieved great success by incorporating phone accuracy in the objective function to be optimized [4]. Later, several different objective functions related to MPE have been proposed, including Minimum Phone Frame Error (MPFE) [5], Minimum Divergence (MD) [6], and a physical-state level version of Minimum Bayes Risk [7], referred to as SMBR here. Some modifications to the objective function and data selection of MPE based on statistics of MPE training process have also been explored [8][9]. The concept of maximum margin successful in machine learning has been recently adopted for discriminative training as well. Large Margin Estimation [10][11] and Soft Margin Estimation [12] for HMM training have achieved very good results on several databases. Boosted-MMI has also been proposed using a similar idea [13].

Povey recently conducted a study comparing MPE to several MPE-related approaches on English and Arabic broadcast news tasks [1]. Here in this paper, we evaluate MPFE, sMBR, and MPE on Mandarin Broadcast news tasks, and investigate several possible modifications to these approaches, as well as their impact on word and character accuracies.

Below in Section 2, we introduce MPE, MPFE, sMBR, and the possible modifications to them. Section 3 explains the experimental settings. Section 4 gives the experimental results and discussions. Concluding remarks are made in Section 5 .

\section{MPE AND MPE-RELATED APPROACHES FOR DISCRIMINATIVE TRAINING}

From a unified point of view, Minimum Phone Error (MPE), Minimum Phone Frame Error (MPFE), and physical-state level version of Minimum Bayes Risk (sMBR) training all have the framework of minimum error training, in which an objective function can be expressed as a form of expected transcription accuracies. Precisely, the objective function has the general form as follows:

$$
f(\lambda)=\sum_{r} \sum_{u \in W^{r}} P_{\lambda}\left(u \mid O_{r}\right) \operatorname{Acc}\left(s_{r}, u\right)
$$

where $P_{\lambda}\left(u \mid O_{r}\right)$ is the posterior probability of the possible hypothesis $u$ from the hypothesis space $W^{r}$ given an acoustic observation $O_{r}$, and the set of model parameters $\lambda$ :

$$
P_{\lambda}\left(u \mid O_{r}\right)=\frac{P_{\lambda}^{\kappa}\left(O_{r} \mid u\right) P(u)}{\sum_{u \in W^{r}} P_{\lambda}^{\kappa}\left(O_{r} \mid u\right) P(u)},
$$


where $\kappa$ is the weighting factor for the acoustic scores, while the accuracy function, $\operatorname{Acc}\left(s_{r}, u\right)$ is to measure how accurate a hypothesis $u$ is compared to the reference $s_{r}$.

\subsection{MPE}

In the case of MPE, the accuracy function is defined as the raw phone accuracy of the hypothesis $u$ given the correct transcript $s_{r}$, which equals to the number of correctly recognized phones minus the number of insertions. The objective function then becomes the expected raw phone accuracy of the training data, or the sum of the raw phone accuracies of all possible hypotheses given the reference, weighted by the likelihood of each hypothesis, as a function of the model [4]:

$$
F_{M P E}(\lambda)=\sum_{r} \frac{\sum_{u \in W^{r}} P_{\lambda}^{\kappa}\left(O_{r} \mid u\right) P(u) \operatorname{Acc}\left(s_{r}, u\right)}{\sum_{u \in W^{r}} P_{\lambda}^{\kappa}\left(O_{r} \mid u\right) P(u)},
$$

where the hypothesis space is approximated as all the hypotheses in the lattice $W^{r}$. In practice, an approximation is used to calculate the raw phone accuracy in a lattice, in which each hypothesized phone $q$ contributes an amount of accuracy, PhoneAcc $(q)$, depending on how much it overlaps in time with the reference phone segments $\mathrm{z}$ as follows:

$$
\text { PhoneAcc }(q)=\max _{z}\left\{\begin{array}{ll}
-1+2 e(q, z) & \text { if } q=z \\
-1+e(q, z) & \text { if } q \neq z
\end{array},\right.
$$

where $e(q, z)$ is the fraction of $z$ segment that overlaps with $q$. The approximated phone accuracy for a whole utterance is then defined as:

$$
\operatorname{Acc}\left(s_{r}, u\right)=\sum_{q \in u} \text { PhoneAcc }(q) \text {. }
$$

\subsection{MPFE and SMBR}

MPFE [5] replaces the phone-by-phone accuracy for MPE as defined in (4) with a frame-by-frame accuracy, which counts the number of frames having correct phone labels in hypothesis s as follows:

$$
\begin{gathered}
\text { PhoneAcc }(q)=\sum_{t=\operatorname{start}(q)}^{\text {end }(q)} \delta\left(q, z\left(s_{r}, t\right)\right) \\
\delta\left(q, z\left(s_{r}, t\right)\right)= \begin{cases}1 & , \text { if } q=z\left(s_{r}, t\right) \\
0 & , \text { if } q \neq z\left(s_{r}, t\right)\end{cases}
\end{gathered}
$$

where $\operatorname{start}(q)$ and end $(q)$ are the start and end time in frames respectively, $z\left(s_{r}, t\right)$ is the phone identity for the reference transcript $s_{r}$ at frame $t, \delta\left(q, z\left(s_{r}, t\right)\right)$ has a value 1 when $q$ is the same as $z\left(s_{r}, t\right)$, and 0 otherwise. This accuracy function basically adds 1 point for each frame with correct hypothesized phone. While the accuracy function in MPE tends to favor deletion over insertion errors (for every hypothesized phone, a "-1" is always introduced into the accuracy as in (4), which leads to a potentially higher accuracy for a hypothesis with more deletions), the one used in (6) for MPFE treats these two types of errors equally.

Like MPFE, sMBR [7] also accumulates accuracy frame by frame to compute the total accuracy of an utterance as in (6). However, sMBR counts the number of frames having a correct state label rather than just having the correct phone label.

\subsection{Variants of MPFE and sMBR}

The accuracy functions used for MPFE and sMBR are essentially the number of frames correctly labeled. Based on this kind of definition, phones of varied length may have been treated unequally during optimization. Moreover, in addition to gaining nothing in accuracy for those frames with errors, a negative penalty might help as well [8].

We explore these ideas by redefining the function $\delta\left(q, z\left(s_{r}, t\right)\right)$ in (6) for MPFE. A negative constant, rather than a zero, can be assigned for a frame with an incorrect phone label as following:

$$
\delta\left(q, z\left(s_{r}, t\right)\right)=\left\{\begin{array}{ll}
1 & , \text { if } q=z\left(s_{r}, t\right) \\
-\rho & , \text { if } q \neq z\left(s_{r}, t\right)
\end{array},\right.
$$

where $\rho$ is the penalty factor.

This can be similarly applied to sMBR. A zero is assigned when the frame has an incorrect state label but is correct in the phone label. If the phone level is not correct either, a negative constant is applied as following:

$$
\delta\left(q, w\left(s_{r}, t\right), z\left(s_{r}, t\right)\right)= \begin{cases}1 & , \text { if } q=w\left(s_{r}, t\right) \\ -\rho & , \text { if } q \neq z\left(s_{r}, t\right) \\ 0 & , \text { otherwise }\end{cases}
$$

where $w\left(s_{r}, t\right)$ is the state identity for the reference transcript $s_{r}$ at frame $t$.

For both MPFE and SMBR, the values of the new $\delta$ function computed as above can be further normalized by the length of the phone in number of frames. The objective functions thus obtained, as variants to the original MPFE and sMBR, are referred to as $\mathrm{MPFE}+$ pen (penalty)+len (length normalization) and sMBR+pen+len, respectively.

\section{EXPERIMENTAL SETTING}

Below we describe the corpus and the baseline recognition system used for the experiments in this paper.

\subsection{Corpus}

The speech data we used for the experiments were taken from the corpus of MATBN (Mandarin Across Taiwan 


\begin{tabular}{|c|c|c|r|}
\hline $\begin{array}{c}\text { Training ap- } \\
\text { proaches }\end{array}$ & $\begin{array}{c}\text { numerator } \\
\text { lattice statis- } \\
\text { tics }\end{array}$ & $\begin{array}{c}\text { denominator } \\
\text { lattice statis- } \\
\text { tics }\end{array}$ & $\begin{array}{c}\text { Estimated I- } \\
\text { smoothing } \\
\text { parameter } \tau\end{array}$ \\
\hline MPE & $1.005032 \mathrm{E}+06$ & $1.005032 \mathrm{E}+06$ & 25.00 \\
\hline MPFE & $8.559231 \mathrm{E}+06$ & $8.559231 \mathrm{E}+06$ & 212.91 \\
\hline MPFE+pen+len & $9.895510 \mathrm{E}+05$ & $9.895510 \mathrm{E}+05$ & 24.61 \\
\hline sMBR & $5.267830 \mathrm{E}+06$ & $5.267830 \mathrm{E}+06$ & 131.04 \\
\hline sMBR+pen+len & $6.580281 \mathrm{E}+05$ & $6.580281 \mathrm{E}+05$ & 16.37 \\
\hline
\end{tabular}

Table 1 Estimation of the I-smoothing parameter $\tau$ based on the numerator and denominator statistics.

Broadcast News), which includes 30 hours of news from 2001, 146 hours from 2002, and 24 hours from 2003, collected and transcribed by a joint project of Academia Sinica and Public Television Service Foundation of Taiwan [14]. 27 hours of gender-balanced speech data from field reporters (6066 pieces of news), together with the corresponding phone alignment information were used here, among which 25.5 hours were used for acoustic model training, while 1.5 hours for testing.

\subsection{Baseline Recognition System}

For baseline acoustic modeling, a set of Hidden Markov Models were trained using HTK based on ML criterion for 112 right-context-dependent INITIAL's, 38 contextindependent FINAL's, and a silence model. Various MPErelated discriminative training approaches were then applied on these ML models. The features are 39-dimensional MFCC coefficients with Cepstral Mean Subtraction (CMS). We used a 3-gram language model trained with a text corpus

(a) 62.00

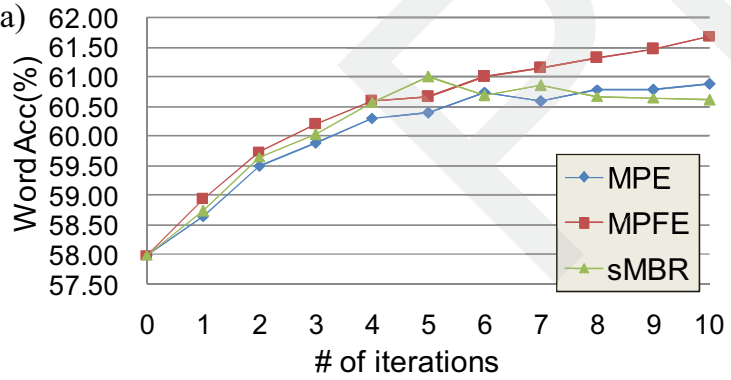

(b) 78.00

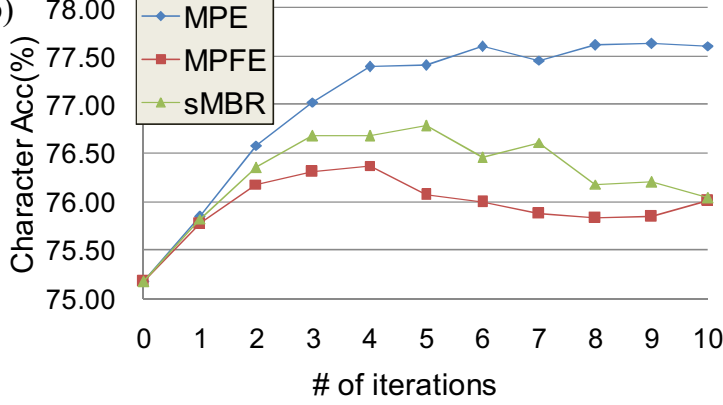

Figure 1: Comparison among MPE, MPFE, and $S M B R$ in terms of (a) word and (b) character accuracies.
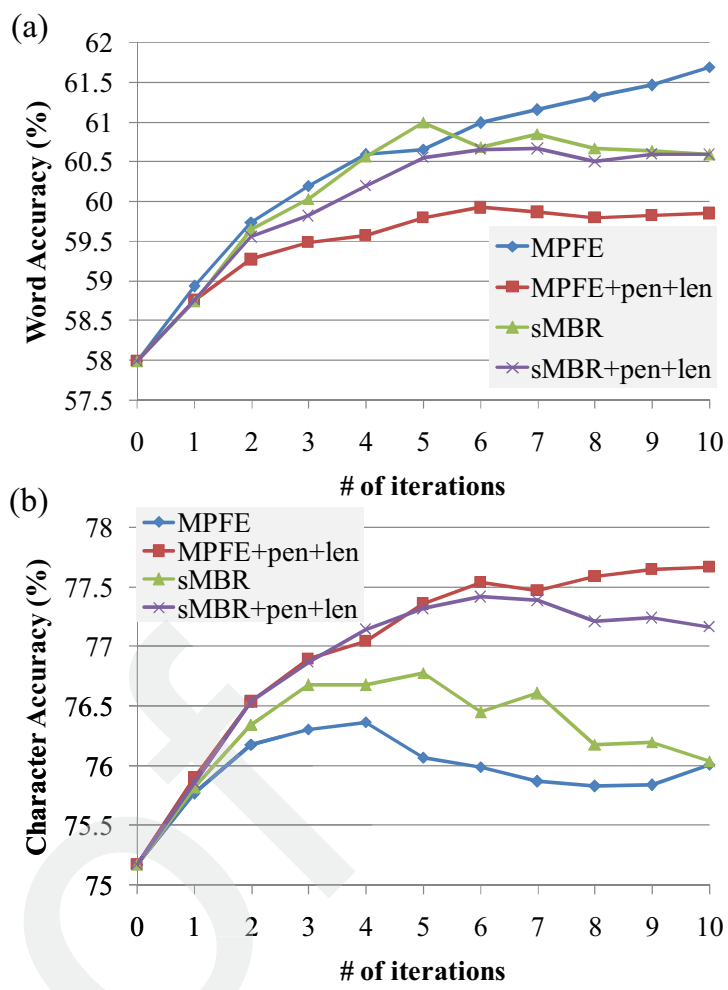

Figure 2: Comparing MPFE and $S M B R$ with their variants in terms of (a) word and (b) character accuracies.

of $170 \mathrm{M}$ Chinese characters provided by Central News Agency (CNA)of Taiwan, collected in 2001 and 2002, with Katz smoothing. The vocabulary has $72 \mathrm{~K}$ words.

\section{EXPERIMENTAL RESULTS AND DISCUSSIONS}

First, we compare the recognition performance of the acoustic models trained from the three MPE-related discriminative training approaches mentioned in Section 2. The numerator and denominator lattices needed for accumulating statistics were generated for training utterances using a 2-gram language model. The performance of these approaches is usually highly sensitive to the choice of the parameter $\tau$ for I-smoothing, which therefore should be carefully selected. In fact, a promising range of better values for $\tau$ can be decided based on the numerator and denominator statistics, as previously suggested [1]. Table 1 lists the relevant statistics and the value of $\tau$ we chose.

In Figure 1, we compare the recognition performance of acoustic models trained by MPE, MPFE, and SMBR, respectively in terms of word accuracy (Figure 1(a)) and character accuracy (Figure 1(b)). The accuracy of the model trained at each iteration is plotted, showing the trend of how the training process converged. Comparing the results from the three approaches, we can see that MPFE gave the best results in word accuracy, while MPE was apparently superior when character accuracy is considered. While the results in word 
(a)

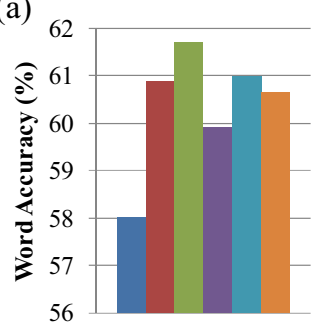

(b) 78

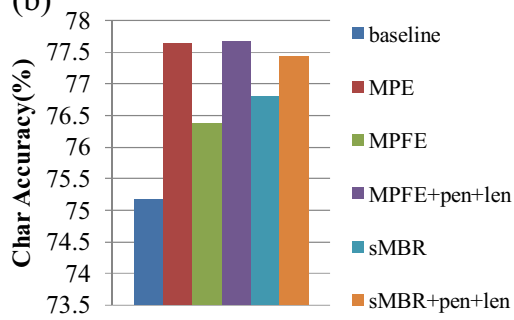

Figure 3: Summary of the best results for all the approaches analyzed here in terms of (a) word and (b) character accuracies.

accuracy in general agree with those found in English and Arabic tasks [1], the trend in character accuracy is in fact quite different, showing that MPE outperformed MPFE and sMBR. This is important for Chinese language, since for Chinese language character accuracy makes better sense than word accuracy.

We further investigated the effect of length normalization (+len) and error penalty (+pen) for MPFE and sMBR training, as mentioned earlier in Section 2.3. Figure 2 compares MPFE and sMBR with their variants, MPFE+pen+len and sMBR+pen+len, again in word accuracy (Figure 2(a)) and character accuracy (Figure 2(b)). In the case of MPFE, introducing error penalties and length normalization improved the character accuracy significantly, with an absolute improvement of $1.3 \%$. However, the same technique seemed to be harmful for word accuracy. On the other hand, sMBR also benefited from the modifications used here when character accuracy was concerned. sMBR+pen+len gave an absolute improvement of $0.64 \%$ in character accuracy, while there was again a slightly decrease in word accuracy. The introduced error penalty and normalization terms generally improved character accuracy probably because erroneous phones here got penalized to a degree proportional to the number of them (in contrast to the original case where the penalties were always zero regardless of the number of phones a word spans). Also, in principle, here each phone contributes the same amount of character accuracy since it has been normalized by length.

\section{CONCLUDION}

In this paper report the results of comparing MPE, MPFE, sMBR and their variants for transcription of large vocabulary Mandarin broadcast news. Figure 3 summarizes the best results, regardless of number of iterations, of all the three approaches and their variants tested. The results show that MPE outperformed MPFE and SMBR in character accuracy. While phone length normalization and error penalty in general degraded the word accuracy for MPFE and SMBR, they improved character accuracy significantly, making MPFE and sMBR compatible to MPE.

\section{REFERENCES}

[1] D. Povey, B. Kingsbury, "Evaluation of Proposed Modifications to MPE for Large Scale Discriminative Training," Proc. ICASSP, 2007.

[2] L. Bahl, P. Brown, P de Souza, R. Merce, "Maximum Mutual Information Estimation of Hidden Markov Model Parameters for Speech Recognition," Proc. ICASSP, 1986.

[3] B.-H. Juang, W. Chou, C.-H Lee, "Minimum Classification Error Rate Methods For Speech Recognition," IEEE Transactions on Speech and Audio Processing, 1997.

[4] D. Povey, P.C. Woodland, "Minimum Phone Error And Ismoothing For Improved Discriminative Training," Proc. ICASSP, 2002.

[5] J. Zheng, A. Stolcke, "Improved Discriminative Training Using Phone Lattices," Interspeech, 2005.

[6] J. Du, P. Liu, F. K. Soong, J.-L. Zhou, R.-H. Wang, "Minimum Divergence Based Discriminative Training," Interspeech, 2006.

[7] M. Gibson, T. Hain, "Hypothesis Spaces for Minimum Bayes Risk Training in Large Vocabulary Speech Recognition," Interspeech, 2006.

[8] S.-H. Liu, F.-H. Chu, S.-H. Lin, B. Chen, "Investigation Data Selection for Minimum Phone Error Training of Acoustic Models," Proc. ICME, 2007.

[9] S.-H. Liu, F.-H. Chu, S.-H. Lin, H.-S. Lee, B. Chen, "Training Data Selection for Improving Discriminative Training of Acoustic Models," Proc. ASRU, 2007.

[10] H. Jiang, X. Li, C. Liu, "Large Margin Hidden Markov Models for Speech Recognition," IEEE Trans. Acoustics, Speech and Signal Processing, Vol.14, No.5, pp. 1584-1595, 2006.

[11] F. Sha, L. K. Saul, "Comparison of Large Margin Training to Other Discriminative Methods for Phonetic Recognition by Hidden Markov Models," ICASSP, 2007.

[12] J. Li, M. Yuan, C.-H. Lee, "Soft Margin Estimation of Hidden Markov Model Parameters," Interspeech, 2006.

[13] D. Povey, D. Kanevsky, B. Kingsbury, B. Ramabhadran, G. Saon, and K. Visweswariah, "Boosted MMI for Model and Feature- Space Discriminative Training," ICASSP, 2008.

[14] H.-M. Wang, B. Chen, J.-W. Kuo, and S.-S Cheng "MATBN: A Mandarin Chinese Broadcast News Corpus," Interational Journal of Computational Linguistics and Chinese Language Processing, 2005. 